\title{
Monitoreo químico -microbiológico del agua de la cuenca alta del río Huaura
}

Chemical-microbiological monitoring of water in the upper Huaura river basin

José del Carmen Cuellar Reyes ${ }^{1}$, Nolverio Chinga Manrique ${ }^{1}$, Nicolás Llanos Huertas ${ }^{1}$, Félix Esteban Airahuacho Bautista ${ }^{2}$, José Legua Cárdenas ${ }^{3}$

\section{RESUMEN}

Objetivo: Monitorear aguas superficiales del río Huaura en su cuenca alta con fines de recuperación ambiental sostenible. Métodos: Investigación descriptiva a nivel explicativo fijando seis estaciones para medir parámetros químicos y microbiológicos del agua. Resultados: en laboratorio portátil, temperaturas del agua de $11,2^{\circ} \mathrm{C}$ a $16,3^{\circ} \mathrm{C} ; \mathrm{pH}$ de 7,7 a 7,9 , solidos totales entre 177 a $227 \mathrm{mg} / \mathrm{l}$, conductividad eléctrica de 278 a $300 \mathrm{mg} / \mathrm{L}$, oxígeno disuelto de 5,4 a 9,4 $\mathrm{mg} / \mathrm{L}$, fosfatos de 0,12 a 0,55 , hierro de 0,44 a 15,68 , nitratos de 0,19 a $0,83 \mathrm{mg} / \mathrm{L}$, cloruros de 22 a $34 \mathrm{mg} / \mathrm{L}$, cobre de 0,11 a 0,40 mg/L, zinc de 0,20 a 0,85 mg/L, carbonato de calcio de 107 a 141 $\mathrm{mg} / \mathrm{L}$; mientras en laboratorios Envirolab y Rovill, temperatura del agua de $19,7^{\circ} \mathrm{C}$ a $21^{\circ} \mathrm{C}$, cromo de 0 a 0,002 mg/L, hierro de 0,18 a 2,95 mg/L, manganeso de 0,03 a 0,19 mg/L, plomo de 0 a 0,006 $\mathrm{mg} / \mathrm{L}$, zinc de 0,028 a $0,097 \mathrm{mg} / \mathrm{L}$, ausencia de cadmio, cobre, plata y mercurio, demanda bioquímica de oxigeno de 0 a $4 \mathrm{mg} / \mathrm{L}$, demanda química de oxígeno de 0 a $20 \mathrm{mg} / \mathrm{L}$, coliformes fecales termo tolerantes de 140 a $33000 \mathrm{NMP} / 100 \mathrm{ml}$ y Coliformes totales de 4,900 a 49000 NMP/100 ml. Conclusiones: los niveles de zinc, hierro, manganeso y arsénico, Coliformes termo tolerante y total sobrepasan los límites establecidos y afectarían el ecosistema del río.

Palabras clave: Carga orgánica, contaminación, metales pesados, monitoreo.

\section{ABSTRACT}

Objective: Monitor surface waters of the Huaura River in its upper basin for the purpose of sustainable environmental recovery. Methods: descriptive research at an explanatory level by setting six stations to measure chemical and microbiological parameters of water. Results: in portable laboratory, water temperatures from $11.2^{\circ} \mathrm{C}$ to $16.3^{\circ} \mathrm{C}$; $\mathrm{pH}$ from 7.7 to 7.9 , total solids between 177 to $227 \mathrm{mg} / \mathrm{L}$, electrical conductivity from 278 to $300 \mathrm{mg} / \mathrm{L}$, dissolved oxygen from 5.4 to $9.4 \mathrm{mg} / \mathrm{L}$, phosphates from 0.12 to 0.55 , iron from 0.44 to 15.68 , nitrates from 0.19 to $0.83 \mathrm{mg} / \mathrm{L}$, chlorides from 22 to $34 \mathrm{mg} / \mathrm{L}$, copper from 0.11 to $0.40 \mathrm{mg} / \mathrm{L}$, zinc from 0.20 to $0.85 \mathrm{mg} / \mathrm{L}$, calcium carbonate from 107 to $141 \mathrm{mg} / \mathrm{L}$; while in Envirolab and Rovill laboratories, water temperature from $19.7^{\circ} \mathrm{C}$ to $21^{\circ} \mathrm{C}$, chromium from 0 to $0,002 \mathrm{mg} / \mathrm{L}$, iron from 0.18 to $2.95 \mathrm{mg} / \mathrm{L}$, manganese from 0,03 to $0.19 \mathrm{mg} / \mathrm{L}$, lead from 0 to $0,006 \mathrm{mg} / \mathrm{L}$, zinc from 0,028 to $0,097 \mathrm{mg} / \mathrm{L}$, absence of cadmium, copper, silver and mercury, biochemical oxygen demand from 0 to $4 \mathrm{mg} / \mathrm{L}$, chemical oxygen demand from 0 to $20 \mathrm{mg} / \mathrm{L}$, thermo-tolerant fecal coliforms from 140 to $33,000 \mathrm{NMP} / 100 \mathrm{ml}$ and total coliforms from 4,900 to 49,000 NMP/100 ml. Conclusions: the levels of zinc, iron, manganese and arsenic, thermo tolerant and total coliforms exceed the established limits and would affect the ecosystem of the river.

Keywords: organic load, pollution, heavy metals, monitoring.

\footnotetext{
${ }^{1}$ Facultad de Ingeniería Pesquera. Universidad Nacional José Faustino Sánchez Carrión. Huacho, Perú

${ }^{2}$ Facultad de Ingeniería Agraria, Industrias Alimentarias y Ambiental. Universidad Nacional José Faustino Sánchez Carrión. Huacho, Perú

${ }^{3}$ Facultad de Ingeniería Química y Metalúrgica, Universidad Nacional José Faustino Sánchez Carrión. Huacho, Perú.
} 


\section{INTRODUCCIÓN}

La calidad del agua está afectada por diversos factores como los usos del suelo, la producción industrial y agrícola, el tratamiento que se le da, antes de ser vertida nuevamente a los cuerpos de agua y a la cantidad misma del agua de los ríos y lagos debido a que de esto depende su capacidad de purificación, su cuantificación científica resulta bastante importante y ésta solución es una estrategia básica en el desarrollo de los fundamentos para el manejo de los recursos hídricos (Álvarez, 2008).

El Monitoreo de la calidad del agua es una herramienta fundamental en el manejo de los recursos de aguas dulces, sin agua dulce en la cantidad adecuada, el desarrollo sostenible de la calidad no será posible (Bartram y Ballance, 1996). La contaminación de los ríos en la actualidad es reportada por muchos estudios tales como la de Kalaivani y Dheenadayalan (2013) quienes al monitorear el rio Cooum (India) concluyen que la concentración de metales pesados en el agua supera los límites prescritos por la OMS.

Con respecto al rio Huaura (Perú), el problema principal que afronta es la presencia de diversos agentes relacionados con las actividades de construcción y minería existentes en la zona constituyéndose en una amenaza contaminante al ser utilizado como recipiente de desechos. Las empresas mineras tienen canchas de relave deficientes que en algunos casos han colapsado con el consiguiente daño ambiental, o por lavado de los mismos que se escurren a las lagunas que alimentan a los ríos, observándose extinción de recursos ictiológicos. De allí la importancia de un monitoreo comunitario del río Huaura para contribuir a su recuperación ambiental para su desarrollo sostenible (Calla, 2010). Si bien es cierto que el problema principal de los ríos está asociado con la minería en constante crecimiento, también las ciudades emplazadas en las riberas de ellos, que vierten aguas servidas y residuos sólidos directamente a los cursos de agua son importantes factores contaminantes (Cuellar y Navarro, 2012).

Se justifica en ese sentido la urgente necesidad de prevenir la contaminación y manejar los peligros ambientales en el río Huaura para que en el futuro no genere problemas ni peligros a la población y a la infraestructura en el entorno del río. En consecuencia, el aporte tecnológico al desarrollo regional de la investigación es encontrar científicamente una correcta gestión ante los peligros de contaminación. El objetivo de la investigación fue monitorear aguas superficiales del río Huaura en su cuenca alta, para contribuir a su recuperación ambiental sostenible con equipos portátiles, y validándolo con determinaciones de un laboratorio certificado comparando con los principales protocolos y normativas de calidad de aguas para riego de vegetales, de consumo humano y efluentes mineros acorde a la Ley de aguas Clase III del Perú y los estándares de la Agencia de Protección Ambiental de los Estados Unidos (EPA).

\section{MATERIAL Y MÉTODOS}

El estudio se realizó en la parte alta de la cuenca del río Huaura, ubicada en la zona este de la Región Lima y geográficamente sus puntos extremos se extienden entre los paralelos $10^{\circ} 27^{\prime \prime}$ y los $11^{\circ} 13^{\prime \prime}$ LS y los meridianos $76^{\circ} 32^{\prime \prime}$ y $77^{\circ} 39^{\prime \prime}$ LO, con una superficie de $4770 \mathrm{~km} 2$, con sus dos sub cuencas hidrográficas, la del río Patón y Quichas que en el paraje Mishimachay forman el río Oyón y al unirse con el río Checras en la zona de Tingo forman el río Huaura, la misma que drena directamente al mar en la Caleta Carquín (Figura 1). Se recolectaron muestras de agua, las que fueron analizadas in situ con el laboratorio portátil Hach Modelo CEL. /890 para análisis físico químico del agua, con colorímetro DR890 y en el laboratorio Envirolab Perú, utilizando la metodología de EPA (2000), la demanda bioquímica de oxígeno, la demanda química de oxígeno, metales pesados, mercurio, Coliformes totales. Se localizaron seis estaciones: confluencia del rio Oyón $(A)$, rio Quichas $(A 1)$; rio Patón (A2), confluencia del rio Huaura $(B)$, rio Churín (B1) y rio Chiuchin (B2), se realizaron muestreos en 8 oportunidades en los meses de agosto 2010, septiembre 2011, octubre 2011, noviembre 2011 en 2 oportunidades y diciembre 2011 en 2 oportunidades. Se midió in situ, temperatura del agua y ambiente, $\mathrm{pH}$, conductividad eléctrica, salinidad, sólidos totales, oxígeno 
disuelto, alcalinidad, fosfatos, fierro, nitratos de bajo y medio rango, cobre, zinc y dióxido de cloro tomando como referencia los límites máximos permisibles (LMPS) para agua potable, riego de vegetales y efluentes mineros y los estándares de la EPA (2000). Muestras de agua tomadas en frascos de vidrio esterilizados y refrigerados, tomadas de las mismas estaciones fueron analizadas para Coliformes totales, termo tolerante y fecal. Para el análisis de los metales pesados, en el mismo laboratorio se tomaron muestras en frascos de plásticos de medio litro preservados con $\mathrm{HNO}_{3} 1: 1$ hasta $\mathrm{pH}<2$. Para la demanda bioquímica de oxigeno (DBO), se tomaron las muestras en frascos de plásticos de 1 litro llenados completamente sin burbujas y guardados en refrigeración, la demanda química de oxigeno (DQO), se tomó las muestras en frascos de plástico de 1 litro preservados con ácido sulfúrico 1:1 hasta $\mathrm{pH}$ $<2$. Todos estos análisis se realizaron en el laboratorio Envirolab y Rovill Ings. Para encontrar las diferencias estadísticas de los resultados del presente estudio con las referencias de instituciones reguladoras se utilizó la prueba t de Student.

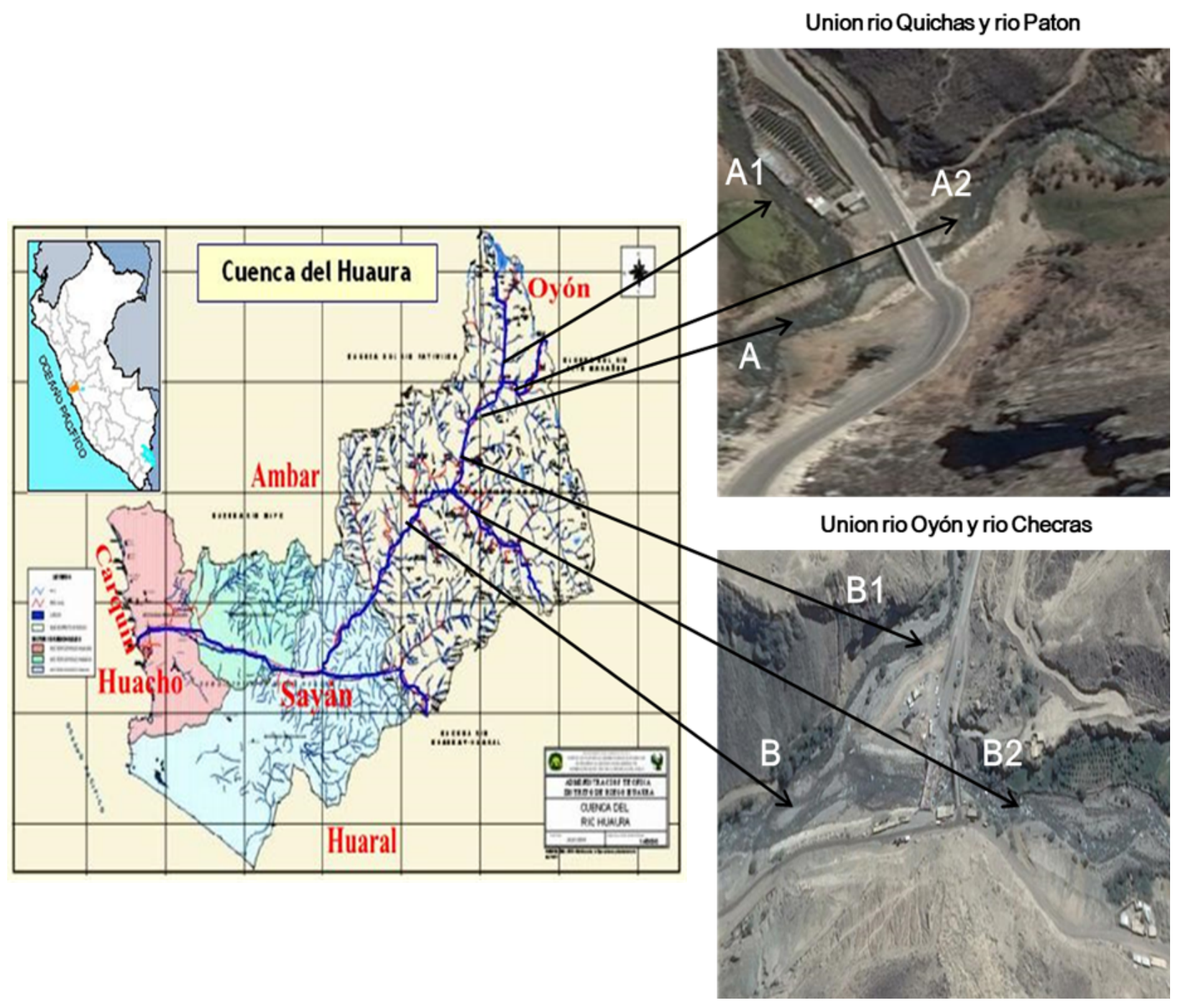

Figura 1. Ubicación de los puntos de muestreo en la cuenca alta del rio Huaura. 


\section{RESULTADOS}

La Tabla 1 muestra los resultados obtenido con el laboratorio portátil. Se registró rangos de temperatura entre $11,3^{\circ} \mathrm{C}$ y $11,5^{\circ} \mathrm{C}$ en las estaciones $\mathrm{A} 1, \mathrm{~A} 2$ y $\mathrm{A}$ ( $3400 \mathrm{msnm}$ ), mientras que en las estaciones $\mathrm{B} 1, \mathrm{~B} 2$ y $\mathrm{B}$ se registró entre $16^{\circ} \mathrm{C}$ y $16,3^{\circ} \mathrm{C}$ (2080 msnm). En ambos puntos de muestreo existe una distancia de 34 $\mathrm{Km}$. En todos los puntos de muestreo, se observó rangos de entre 7,7 y 7,9 de pH, 177,2 a $227,3 \mathrm{mg} / \mathrm{L}$ de solidos totales; 277,50 a $299,83 \mathrm{mg} / \mathrm{L}$ de conductividad eléctrica; 5,44 a $9,43 \mathrm{mg} / \mathrm{L}$ de oxígeno disuelto; 0,12 a 0,55 $\mathrm{mg} / \mathrm{L}$ de fosfatos; 0,44 a $15,68 \mathrm{mg} / \mathrm{L}$ de $\mathrm{Fe}$; 0,19 a $0,83 \mathrm{mg} / \mathrm{L}$ de nitratos; 22,03 a 34,05 $\mathrm{mg} / \mathrm{L}$ de cloruro; 0,11 a $0,40 \mathrm{mg} / \mathrm{L}$ de $\mathrm{Cu} ; 0,20$ a $0,85 \mathrm{mg} / \mathrm{L}$ de $\mathrm{Zn}$ y 106,76 a $141,20 \mathrm{mg} / \mathrm{L}$ de carbonato de calcio.

Tabla 1. Resumen del monitoreo de la cuenca alta de las aguas del río Huaura determinado en laboratorio portátil.

\begin{tabular}{|c|c|c|c|c|c|c|c|c|c|}
\hline \multirow{3}{*}{ Parámetros } & \multicolumn{9}{|c|}{ Puntos de muestreo } \\
\hline & \multirow{2}{*}{ Unidad } & \multicolumn{2}{|c|}{ LMP $^{1}$ del agua } & \multirow{2}{*}{$\begin{array}{c}\text { A1: } \\
\text { Quichas }\end{array}$} & \multirow{2}{*}{$\begin{array}{l}\text { A2: } \\
\text { Patón }\end{array}$} & \multirow{2}{*}{$\begin{array}{c}\text { A: } \\
\text { Oyón }\end{array}$} & \multirow{2}{*}{$\begin{array}{l}\text { B1: } \\
\text { Churín }\end{array}$} & \multirow{2}{*}{$\begin{array}{c}\text { B2: } \\
\text { Checras }\end{array}$} & \multirow{2}{*}{$\begin{array}{c}\text { B: } \\
\text { Huaura }\end{array}$} \\
\hline & & Potable & Riego & & & & & & \\
\hline Temperatura & ${ }^{\circ} \mathrm{C}$ & & & 11,5 & 11,3 & 11,2 & 16,0 & 16,0 & 16,3 \\
\hline $\mathrm{pH}$ & & $6,5-8,5$ & $6,5-8,5$ & 7,7 & 7,8 & 7,8 & 7,9 & 7,9 & 7,9 \\
\hline STD & $\mathrm{mg} / \mathrm{L}$ & 1000,000 & $10-500$ & 180,3 & 177,2 & 180,5 & 188,2 & 227,3 & 208,2 \\
\hline CE & $\mathrm{mg} / \mathrm{L}$ & 1500,000 & 1100,000 & 296,67 & 277,50 & 281,50 & 295,50 & 292,00 & 299,83 \\
\hline OD & $\mathrm{mg} / \mathrm{L}$ & $3 \geq 6$ & $3 \geq 4$ & 6,08 & 5,75 & 5,44 & 9,43 & 8,93 & 9,23 \\
\hline $\mathrm{PO}_{4}^{3-}$ & $\mathrm{mg} / \mathrm{L}$ & $0,01-0,1$ & $0,01-1^{b}$ & $0,25^{\mathrm{a}}$ & $0,12^{\mathrm{a}}$ & $0,21^{\mathrm{a}}$ & $0,43^{\mathrm{a}}$ & $0,22^{\mathrm{a}}$ & $0,55^{\mathrm{a}}$ \\
\hline $\mathrm{Fe}$ & $\mathrm{mg} / \mathrm{L}$ & $0,3-1$ & $1-3,5^{b}$ & 0,63 & 0,44 & 0,84 & $15,68^{\mathrm{a}}$ & 0,75 & $1,48^{\mathrm{a}}$ \\
\hline $\mathrm{NO}_{3}-\mathrm{N}$ & $\mathrm{mg} / \mathrm{L}$ & $0,1-3$ & $5-10$ & 0,67 & 0,19 & 0,30 & 0,25 & 0,24 & 0,83 \\
\hline $\mathrm{CL} \mathrm{O}_{2}$ & $\mathrm{mg} / \mathrm{L}$ & $5-250$ & $100-700$ & 24,40 & 24,52 & 28,53 & 22,03 & 24,17 & 34,05 \\
\hline $\mathrm{Cu}$ & $\mathrm{mg} / \mathrm{L}$ & 1 & $0,2-0,5$ & 0,27 & 0,11 & 0,20 & 0,32 & 0,40 & 0,21 \\
\hline $\mathrm{Zn}$ & $\mathrm{mg} / \mathrm{L}$ & 3 & $0,5-25$ & 0,20 & 0,64 & 0,45 & 0,71 & 0,63 & 0,85 \\
\hline $\mathrm{Ca} \mathrm{CO}{ }_{3}$ & $\mathrm{mg} / \mathrm{L}$ & 500 & $5-300$ & 141,20 & 134,96 & 111,40 & 137,20 & 112,20 & 106,76 \\
\hline
\end{tabular}

${ }^{1}$ LMP: Límite máximo permitido por ley de aguas Ministerio del Ambiente. (2008).

La Tabla 2 muestra los resultados obtenido en los laboratorios Envirolab y Rovill, quienes registraron, en ambas estaciones, entre $21 \mathrm{y}$ $21,7^{\circ} \mathrm{C}$ de temperatura ambiental; 19,70 y 21 , ${ }^{\circ} \mathrm{C}$ en el agua; ausencia de cadmio, cobre, plata y mercurio; 0 a 0,002 de $\mathrm{Cr}, 0,182$ a
2,950 de Fe; 0,03 a $0,190 \mathrm{Mn}, 0$ a $0,006 \mathrm{~Pb}$; 0,028 a $0,097 \mathrm{Zn} ; 0$ a $4 \mathrm{mg} / \mathrm{L}$ de DBO; 0 a 20 $\mathrm{mg} / \mathrm{L} \mathrm{DQO} ; 140$ a $33000 \mathrm{NMP} / 100 \mathrm{ml}$ de Coliformes fecales termo tolerantes y 4,900 a 49,000 NMP/100 ml de Coliformes totales. 


\begin{tabular}{|c|c|c|c|c|c|c|c|c|}
\hline \multirow[b]{2}{*}{ Parámetros } & \multicolumn{8}{|c|}{ Puntos de muestreo } \\
\hline & Unidad & $\begin{array}{c}\text { Límite } \\
\text { permisible }\end{array}$ & $\begin{array}{c}\text { A1: } \\
\text { Quichas }\end{array}$ & $\begin{array}{c}\text { A2: } \\
\text { Patón }\end{array}$ & $\begin{array}{l}\text { A: } \\
\text { Oyón }\end{array}$ & $\begin{array}{c}\text { B1: } \\
\text { Churín }\end{array}$ & $\begin{array}{c}\text { B2: } \\
\text { Checras }\end{array}$ & $\begin{array}{c}\text { B: } \\
\text { Huaura }\end{array}$ \\
\hline Temperatura agua & ${ }^{\circ} \mathrm{C}$ & & & & & 20,00 & 21,00 & 19,70 \\
\hline $\begin{array}{l}\text { Temperatura } \\
\text { ambiental }\end{array}$ & ${ }^{\circ} \mathrm{C}$ & & & & & 21,000 & 21,500 & 21,700 \\
\hline $\mathrm{Cd}$ & $\mathrm{mg} / \mathrm{L}$ & 0,001 & $0^{2}$ & 0 & 0 & 0 & 0 & 0 \\
\hline $\mathrm{Cr}$ & $\mathrm{mg} / \mathrm{L}$ & 0,001 & 0 & 0 & 0 & 0 & 0,002 & 0,002 \\
\hline $\mathrm{Cu}$ & $\mathrm{mg} / \mathrm{L}$ & 0,002 & 0 & 0 & 0 & 0 & 0 & 0 \\
\hline $\mathrm{Fe}$ & $\mathrm{mg} / \mathrm{L}$ & $0,003^{b}$ & $0,844^{a}$ & $0,182^{\mathrm{a}}$ & $0,863^{\mathrm{a}}$ & $0,5^{\mathrm{a}}$ & $0,640^{a}$ & $2,950^{a}$ \\
\hline $\mathrm{Mn}$ & $\mathrm{mg} / \mathrm{L}$ & $0,001^{b}$ & $0,101^{a}$ & $0,03^{\mathrm{a}}$ & $0,097^{a}$ & $0,077^{\mathrm{a}}$ & $0.130^{a}$ & $0,190^{a}$ \\
\hline $\mathrm{Pb}$ & $\mathrm{mg} / \mathrm{L}$ & $0,001^{b}$ & 0,001 & 0 & 0 & $0,002^{\mathrm{a}}$ & $0,002^{\mathrm{a}}$ & $0,006^{a}$ \\
\hline $\mathrm{Zn}$ & $\mathrm{mg} / \mathrm{L}$ & $0,002^{b}$ & $0,031^{a}$ & $0,028^{a}$ & $0,051^{a}$ & $0,032^{\mathrm{a}}$ & $0,042^{\mathrm{a}}$ & $0,097^{a}$ \\
\hline $\mathrm{Ag}$ & $\mathrm{mg} / \mathrm{L}$ & 0,002 & 0 & 0 & 0 & 0 & 0 & 0 \\
\hline $\mathrm{Hg}$ & $\mathrm{mg} / \mathrm{L}$ & 0,0001 & 0 & 0 & 0 & 0 & 0 & 0 \\
\hline DBO5 & $\mathrm{ml} / \mathrm{l}$ & 1 & 0 & 0 & 0 & 4 & 0 & 0 \\
\hline DQO & $\mathrm{mg} / \mathrm{L}$ & 20 & 0 & 0 & 0 & 20 & 0 & 0 \\
\hline Coliformes fecales & $\mathrm{NNP} / 10$ & $\mathrm{ml}$ & & & & & & \\
\hline Termo tolerantes & & $0^{\mathrm{b}}$ & $33000^{a}$ & $140^{a}$ & $23000^{a}$ & $25000^{a}$ & $25000^{a}$ & $12450^{a}$ \\
\hline Totales & & $0^{\mathrm{b}}$ & $33000^{a}$ & $4900^{a}$ & $23000^{a}$ & $49000^{a}$ & $49000^{a}$ & $12500^{a}$ \\
\hline
\end{tabular}

\footnotetext{
EPA: Agencia de protección ambiental de EUA

${ }^{2}$ N.D: no determinado
}

\section{DISCUSIÓN}

Los resultados de las temperaturas registradas en todas las estaciones, indican una relación inversamente proporcional entre la temperatura y la altura sobre el nivel del mar como lo señala Jesús et al.(2008); a mayor altura hay menor presión atmosférica, la temperatura disminuye a un grado por cada cien metros de ascenso y al contrario en las profundidades marinas aumenta en base a lo anterior. Las aguas del río cumplen con los requisitos mínimos para la vida acuática y la recreación para esta zona. Ahmed et al., (2013) determina que el factor de bioacumulación de los metales pesados, especialmente $\mathrm{Zn}$, Fe y $\mathrm{Cu}$, fue mayor en la temporada de aumento de la temperatura lo que hay que tomar en cuenta dada la presencia de metales pesados en la zona de estudio. La conductividad eléctrica registrada; de 277,50 a $299,83 \mathrm{mg} / \mathrm{L}$ y por DIGESA(2001) de 115,5 a $478 \mathrm{~ms} / \mathrm{Sm}$ no revisten contaminación. En $\mathrm{pH}$, los valores registrados de 7,7 a 7,9 tienden a rangos neutros cercanos a alcalinos. En cambio, los hallados por DIGESA (2000) reporta valores de 5,18 a 8,01 lo que tienden a ácido y a la vez a alcalino en las estaciones cercanas a Churín y Picoy, puede deberse a la planta de filtrado de minerales de la empresa minera Izcaycruz.

El nivel de oxígeno disuelto, se encuentran en un rango de 5,44 a 9,43 mg/L. Hay que tener en cuenta que por debajo de $5 \mathrm{mg} / \mathrm{L}$ están cercanas a la hipoxia es decir las consecuencias son desaparición de organismos aerobios y los que están por encima de 12 se encuentran en condición de sobresaturada cuyas consecuencias serían fatales.

Los carbonatos registrados de 106,76 a $141,20 \mathrm{mg} / \mathrm{L}$, indica alcalinidad aceptable. El agua usualmente presenta iones carbonatos $(\mathrm{CO} 3=)$ y bicarbonatos (HCO3-), asociados con los cationes $\mathrm{Na}^{+}, \mathrm{K}^{+} \mathrm{Ca}^{+2} \mathrm{y} \mathrm{Mg}^{+2}$, (Rivera, et al., 2004). De los registros realizados en el estudio nos arrojan un resultado no muy alentador para la crianza de truchas, en el mes 
octubre el contenido de carbonatos es apto para la crianza de peces, mientras que en los meses de noviembre y diciembre se registraron niveles de carbonatos elevado, no apto para la crianza de truchas. Las truchas soportan mal las variaciones bruscas de $\mathrm{pH}$. La estabilidad viene dada por la presencia de lo que se conoce como reserva alcalina o sistema tampón, que depende de la concentración en las aguas de carbonato y bicarbonato (Rivera et al., 2004. Las aguas bicarbonatadas sódicas son malas para riego, debido a la fijación del sodio $\left(\mathrm{Na}^{+}\right)$en el terreno y creación de un medio alcalino. Los niveles de fosforo resultaron altos. Las causas del alto contenido de fosfatos en las aguas del rio se encuentran en las aguas residuales que se vierten directamente a las aguas superficiales provenientes de fertilizantes eliminados del suelo por el agua o el viento, excreciones humanas y de animales, detergentes y productos de limpieza. (Rivera et al., 2004).

Los nitratos fluctuaron de 0,0 a 5,2 mg/L., por lo que pueden formar nitrosaminas, nitrosamidas y compuestos que favorecen cuadros cancerígenos. (Rivera et al., 2004). Las concentraciones de cobre entre 0,04 a $0,58 \mathrm{mg} / \mathrm{L}$, son valores ligeramente altos.

El zinc no se considera tóxico, aunque confiere al agua un sabor desagradable, en aguas saladas y dulces es muy estable (Rivera et al., 2004). Los resultados en los laboratorios certificados para zinc fueron de 0,07 a 1,52 mg/L, valores por encima de los estándares de la EPA. Altas concentraciones de zinc reducen el crecimiento de la planta y provoca acumulaciones indeseables en los tejidos. Se acumula irreversiblemente el zinc en el suelo, por lo que los excesos pueden llegan a contaminar los suelos (Rivera et al., 2004), los cuales pueden convertirse en suelos no productivos o producir cosechas inaceptables.

El zinc es esencial en la síntesis de DNA, en procesos implicados en la inmunidad, curación de las heridas, percepción del sentido del gusto, síntesis de esperma y el desarrollo físico normal, lo que resulta sumamente peligroso por los resultados hallados que sobrepasan el límite máximo establecido por la EPA. Las estaciones en los ríos que presentan los niveles más altos son los de Patón, Oyón, Churín, Chiuchin, Huaura sobrepasando los LMP para uso potable, de riego de vegetales y de efluentes mineros, el rio Quichas solo sobrepasa para usos potables los cuales sugieren su origen en la actividad minera, en cuanto al $\mathrm{Fe}$ en las estaciones B1 y B, todos los valores sobrepasan los límites establecidos por la EPA, las mismas puede depositarse como hidróxido y obturar las branquias de los organismos, disminuyendo su potencial respiratorio, los huevos y las larvas cultivadas son más sensibles a estos metales, produciéndose entonces su muerte, (Rivera et al., 2004). El Fe en el medio acuático no es nocivo al estar en bajo contenido, pero suele serlo en presencia de altas concentraciones, para las truchas, con valores de $\mathrm{pH}$ en aguas de 6,5 a 7,5 y concentraciones de 0,9 ppm de hierro, es de efecto mortal (Rivera et al., 2004).

El cromo total en la Estación B2 y B sobrepasa el límite establecido por la EPA. Estas cantidades excesivas pueden reducir el crecimiento y provocar acumulaciones indeseables en los tejidos (Reyes, 2016). En el agua los niveles naturales de $\mathrm{Cr}$ son bajos alcanzando en los ríos concentraciones que varían entre 0,1 a $5 \mathrm{mg} / \mathrm{L}$. La Agencia Internacional para la Investigación de Cáncer (IARC) clasifica a los compuestos de $\mathrm{Cr}$ como cancerígenos conocidos. En cuanto al $\mathrm{Mn}$, los resultados sobrepasan el límite establecido por la EPA (2001). El exceso de Mn puede causar síntomas de toxicidad y deficiencia en plantas. Concentraciones altamente tóxicas de Manganeso causan inflamaciones de las paredes celulares, abrasamiento de las hojas y puntos marrones en las hojas, en cantidades excesivas reducen el crecimiento y provocan acumulaciones indeseables en los tejidos (Reyes 2016). En PB, los resultados indican que existe contaminación de las aguas. El envenenamiento por $\mathrm{Pb}$ causa una serie de efectos adversos para la salud humana, pero es particularmente perjudicial para el desarrollo neurológico de los niños. En DQO se registra valor alto en la Estación $B 1$, que corresponde a la zona de Churín. En DBO, en la Estación B1, se registra $4 \mathrm{mg} / \mathrm{L}$ valor por encima del límite establecido por la EPA y se relaciona con los resultados de la presencia de Coliformes, ya que la materia orgánica se 
acumula en las raíces o extremidades de los vegetales, y los afectados son los consumidores que los ingieren directamente.

La putrefacción de la materia orgánica en el agua produce una disminución de la cantidad de oxígeno. Los Indicadores microbiológicos, presentan concentraciones sumamente altas, en todas las estaciones muestreadas se superaron los valores máximos de Coliformes totales convirtiéndolas en agua no apta para actividades con contacto directo y para riego de frutas y hortalizas (DIGESA 2001). Hay que tener en cuenta que la contaminación por microorganismos puede acarrear graves problemas no solamente a la salud de las plantas y animales sino también a la del hombre, consumidor de ellos, los resultados alcanzan niveles exorbitantes de Coliformes totales en la Cuenca Alta del rio Huaura. Hong et al., (2014) señalan que los metales pesados son contaminantes importantes en las aguas superficiales y representan peligros ambientales persistentes que pueden perjudicar seriamente la salud humana y ecológica. Sarah et al., (2014) sugieren que los análisis de metales pesados son indicadores importantes para la toma de decisiones involucrados en la gestión del riesgo. Kalaivani y Dheenadayalan (2013) al evaluar la calidad del agua en el río Cooum con respecto a su concentración de metales pesados encontraron niveles elevados de $\mathrm{Cr}$, $\mathrm{Zn}, \mathrm{Cu}$ y Fe que exceden los límites prescritos por la Organismo Mundial de la Salud, y que afectarían la salud de una población que aumenta anualmente de forma exponencial, y que a la vez conduciría a la generación de gran cantidad de aguas residuales vertidas directamente a la cuenca. Similar situación fue observada en la presente investigación, donde los resultados obtenidos muestran que el agua del rio Huaura contiene niveles superiores, en fosfato, $\mathrm{Fe}, \mathrm{Mn}, \mathrm{Pb}$ y $\mathrm{Zn}$, con respecto a los recomendados para el agua con fines de consumo humano. Asimismo, se observó altas concentraciones de Coliformes fecales termotolerantes y Coliformes fecales totales, que se relacionan con el nulo tratamiento de las aguas servidas de las poblaciones que crecen alrededor de la cuenca del rio Huaura.

\section{AGRADECIMIENTOS}

La investigación fue financiada por el Fondo Camisea (FOCAM), gestionado por el Vicerrectorado de Investigación de la Universidad Nacional José Faustino Sánchez Carrión, y ejecutada a través del proyecto de investigación "Monitoreo comunitario para la recuperación ambiental sostenible de la cuenca alta del rio Huaura".

\section{REFERENCIAS BIBLIOGRÁFICAS}

Álvarez, H. Aurelia Fidiligno Niño. (2008) Guía de investigación. USTA. Bogotá, 1968.

Ahmed Th. A. Ibrahim, Hossam M. Omar (2013). Seasonal variation of heavy metals accumulation in muscles of the African Catfish Clarias gariepinus and in River Nile water and sediments at Assiut Governorate, Egypt. Journal of Biology and Earth Sciences, 201 3, Vol 3, Issue 2, B236.

Bartram, J. \& R. Ballance. (1996). Water Quality Monitoring - A Practical Guide to the Design and

Implementation of Freshwater Published on behalf of United Nations Environment Programme and the World Health Organization (C) 1996 UNEP/WHO ISBN 0419223207 (Hbk) 041921730. Londres SE1 8HN, Reino Unido.

Calla, Llontop. (2010). Calidad del agua en la Cuenca del rio Rímac afectadas por las actividades mineras. Tesis. UNMSM río Rímac, Sector San Mateo.

Cuellar y Navarro (2012). Niveles de Contaminantes que afectan la calidad de aguas del rio Huaura en su cuenca baja. Universidad Nacional José Faustino Sánchez Carrión.

Dirección General de Salud Ambiental DIGESA (2001) Informe $\mathrm{N}^{\circ} 13$-2002USA. Monitoreo del Río Huaura. Dirección Ejecutiva de Salud Ambiental. Resultados del Monitoreo 2000-2001.

EPA. (2001). Concentrations of Lead in Blood. U.S. Environmental Protection Agency. http:/yosemite.epa.gov/OCHP/OCHP.

Jesús, P.A. Álvarez Juan E.R. Panta, Carlos R. Ayala y Elizabeth H. Acosta (2008). Calidad Integral del Agua Superficial en la Cuenca Hidrológica del Río Amajac. 
Colegio de Postgraduados Campus Montecillo, Km 36.5 Carretera MéxicoTexcoco, Estado de México, C. P. 56230 Información Tecnológica-Vol. 19 $\mathrm{N}^{\circ} 6-2008, \quad$ pá g.: $21-32$ doi:10.1612/inf.tecnol.3975it.07.

Kalaivani T.R. and M.S. Dheenadayalan T.R. (2013). International Research Journal of Environment Seasonal fluctuation of Heavy Metal Pollution in Surface water International Science Congress Association ISSN 2319-1414 Vol. 2 (12), 66-73.

Hong, Xin Qian, Hailong Gao, Yulei Wang, \& Bisheng Xia (2014) Seasonal and Spatial Variations of Heavy Metals in Two Typical Chinese Rivers: Concentrations, Environmental Risks, and Possible Sources. State Key Laboratory of Pollution Control and Resource Reuse, School of the Environment, Nanjing University, Nanjing 210023, China; School of Geography, Nantong University, Nantong China Int. J. Environ.

Ministerio del Ambiente. (2008). Estándares nacionales de Calidad Ambiental para Agua. Norma Legal. Lima. DECRETO SUPREMO $\mathrm{N}^{\circ}$ 002-2008-MINAM el peruano 31 de Julio 2008 paginas 377222-377227.

Reyes et al., (2016). Contaminación por metales pesados: Implicaciones en salud, ambiente y seguridad ambiental. Pontificia Universidad Javeriana Bogotá Colombia.

Rivera, N.R, Encina F. Muñoz-Pedreros A. y Mejias. P (2004). La Calidad de las aguas en los Ríos Cautín e Imperial, IX Región-Chile. Información Tecnológica-Vol. 15 N$^{\circ}$ 5-2004, págs..: 89-101.

Sarah F. L. Lynch, Lesley C. Batty 1 and Patrick Byrne (2014). Environmental Risk of Metal Mining Contaminated River Bank Sediment at Redox-

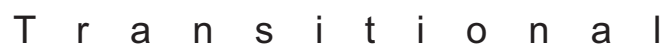
Zones.www.mdpi.com/journal/mineral $\mathrm{s}$.

Correo electrónico:

josecuellarreyes@yahoo.com.pe

Revisión de pares:

Recibido: 07-01-2019

Aceptado: 04-06-2019 